\title{
Clinically Relevant Variations of Recurrent Laryngeal Nerve
}

\author{
Dr. Takkallapalli Anitha ${ }^{1}$, Dr. Dattatray Dombe ${ }^{2}$, Dr. Puli. Dharmendra ${ }^{3}$ \\ ${ }^{1,2}$ Associate Prof.of Anatomy, \\ ${ }^{3}$ Assistant prof.of Anatomy
}

\begin{abstract}
There are multiple variations of recurrent laryngeal nerve in relation to inferior thyroid artery and ligament of Berry which are important from a surgeon's point of view during thyroid surgeries

Aim: The study aims to describe the clinically relevant variations of recurrent laryngeal nerve with respect to inferior thyroid artery and ligament of Berry in the cadavers.

Material and Methods: 108 preserved cadavers (216 sides) were dissected within a span of 5 years i.e from 2008 to 2013. The position of recurrent laryngeal nerve in relation to inferior thyroid artery and ligament of Berry was noted and tabulated.

Results: Our study revealed that in 59\% of cadavers, the recurrent laryngeal nerve passed dorsal to inferior thyroid artery, $30 \%$ of cadavers, it coursed ventral to the artery, followed by $11 \%$ of cadavers where the nerve traversed through a loop formed by the branches of inferior thyroid artery. In $63.8 \%$ of cadavers, the nerve passed superficial to ligament of Berry, in $25 \%$ of cadavers the nerve passed through the ligament, followed by $11.1 \%$ of cadavers where the recurrent laryngeal nerve coursed deep to the ligament.

Conclusion: A thorough knowledge of the laryngeal nerves and their anatomical variations is necessary for safe thyroid surgery.
\end{abstract}

Key Words: Recurrent laryngeal nerve, inferior thyroid artery, anatomical variations, ligament of Berry.

\section{Introduction}

The thyroid is the largest and highly vascular endocrine gland. It is intimately related to two important nerves that control the voice, the superior laryngeal nerve and recurrent laryngeal nerve. The safety of thyroid surgeries mainly depends on complete anatomical knowledge of the arteries and nerves related to it and their anatomical variations. The surgeon who operates on the thyroid should keep in mind the unpleasant morbidity caused by inadvertent injury to the vessels and nerves during surgery.

Galen was the first to describe the recurrent laryngeal nerve (RLN) as a branch of cranial nerve [1]. RLN as a branch of vagus becomes apparent by the end of sixth week. This branch of vagus nerve is associated with the sixth branchial arch of the embryonic pharynx, and it passes directly to the larynx. The course of RLN is determined by the pattern of development of arteries with which it becomes related, and the variations of this pattern will determine variations in the anatomical disposition of this nerve [2].

The RLN originates from the vagus and on the right side, surrounds the right subclavian artery , and on the left, surrounds the aortic arch. Before reaching the larynx, it ascends in the tracheo oesophageal groove in most of the cases and during its course it crosses the inferior thyroid artery [ITA] [3]

The ligament of Berry [LOB] attaches the posteromedial aspect of the thyroid gland and to the sides of cricoid cartilage and first two tracheal rings. The RLN is embedded close to the posterior portion of Berry's ligament and is prone for injury when this ligament is sectioned in order to free the gland from its attachment

This study is undertaken to establish the variations of RLN in relation to ITA and LOB; as the RLN is one of the most frequent and important causes of morbidity in thyroidectomies.

\section{Materials And Methods:}

Collection of specimens: 108 embalmed (216 sides), formalin preserved cadavers between the age group of 40 to 60 years were collected over a period of 5 years i.e 2008 to 2013 in the department of Anatomy, chalmeda Anand Rao Institute of Medical Sciences, Bommakal, Karimnagar, Telangana state, India.

Dissection method was employed for this study. The infrahyoid group of muscles were identified and reflected. The thyroid gland was exposed. The ITA was identified, cleared and the RLN was traced. The relationship of RLN, ITA and LOB was observed and tabulated.

Observation And Results: The following criteria are taken into consideration during dissection and the observations are noted and tabulated on the basis of:-

i). Course of RLN in relation to the ITA

ii). Relationship of the RLN with respect to LOB 


\begin{tabular}{|l|}
\multicolumn{1}{|c|}{ Table No.1--Course of RLN in relation to ITA } \\
\begin{tabular}{|l|cc|cc|l|}
\hline \multicolumn{2}{|c|}{ Right } & \multicolumn{2}{c|}{ Left } & Total \\
& $\mathrm{N}$ & $(\%)$ & $\mathrm{N}$ & $(\%)$ & n- $216 \%$ \\
\hline RLN ventral to ITA & 40 & 37 & 24 & 22 & 30 \\
\hline RLN dorsal to ITA & 50 & 46 & 78 & 72 & 59 \\
\hline $\begin{array}{l}\text { RLN beetween } \\
\text { branches of ITA }\end{array}$ & 18 & 17 & 6 & 6 & 11 \\
\hline Total & 108 & 100 & 108 & 100 & 100 \\
\hline
\end{tabular}
\end{tabular}

Table No.2--Relationship of RLN to the LOB N=108

\begin{tabular}{|l|l|l|}
\hline Position & N & $\%$ \\
\hline RLN superficial to LOB & 69 & 64 \\
\hline RLN deep to the LOB & 12 & 11 \\
\hline RLN through the LOB & 27 & 25 \\
\hline
\end{tabular}

\section{Results}

The position of RLN with respect to ITA on both sides was analyzed. Out of the 216 sides dissected, the RLN coursed dorsal to ITA in 59\% of cadavers, ventral to ITA in 30\% of cadavers followed by $11 \%$ wherein the nerve traversed through the loop formedby branches of ITA. On the right side, the RLN in $48 \%$ of cadavers coursed dorsal to ITA, followed by $37 \%$ of cadavers where the nerve passed ventral to ITA. In $17 \%$ of cadavers the RLN traversed through the branches of ITA. On the left side, the RLN passed dorsal to the ITA in $72 \%$ of cadavers, ventral to ITA in $22 \%$ of cadavers and in $6 \%$ it passed through the loop formed by branches of ITA. [Fig. No.1]

Out of the 108 cadavers dissected, the RLN traversed superficial to LOB in $64 \%$ of cadavers, then in $25 \%$ of cadavers the RLN passed through the ligament of Berry and in least number of cadavers i.e in $11 \%$, the nerve passed deep to the LOB.

\section{Discussion}

Recurrent laryngeal nerves are closely related to the inferior vascular pedicle of thyroid gland. i.e ITA. Injuries involving recurrent laryngeal nerves are more sinister in nature and can cause morbidities ranging from aspiration to stridor. At present the exposure of RLN is mandatory in all thyroid surgeries.

The RLN has significant but varying relationship with the ITA. The knowledge of the relationship between the RLN and ITA is also important for the exteriorization of the thyroid lobe[3]. Simon, after the dissection of 86 nerves in 43 corpses, affirmed that the usual relationship is the one in which the RLN establishes by passing posterior to the ITA. The anterior orientation of the nerve in relation to the artery was occasional, while its position between the arterial branches was a rare finding [4]. Lahey, based on surgical findings, had a similar opinion [5]. Our present study coincides with the above findings [Table No.1].

Considering the right and left sides separately, differences appear. Lekacos et al studied 109 nerves and observed that in 52 cases the right RLN passed posterior to right ITA, followed by 38 cases where the right RLNs traversed through the branches of ITA and in 19 cases the right RLN coursed anterior to right ITA [6]. Hunt et al studied 74 right recurrent laryngeal nerves and observed that the right RLN passed posterior to Right ITA in 40 cases, followed by anterior course of Right RLN in 28 cases and in 6 cases, the right recurrent laryngeal nerves intermingled with branches of right ITA [7]. Our study coincides with the observations made by Hunt et al. [Table No.1].

Berlin et al observed that, out of the 70 Left Recurrent laryngeal nerves dissected, 44 passed dorsal to left ITA, 17 passed ventral to left ITA, followed by least number i.e in 9 cases, the Left RLN passed between the branches of left ITA [8]. The above finding also coincided with the study of Bachhuberetal [9]. Our present study also observed similar finding [Table No.1].

Ligament of Berry anchors the thyroid gland to the laryngotracheal complex [10]. It also corresponds to the area where the RLN is most commonly injured [11]. In a study conducted by P.V. Pradeep et al, out of the 584 RLN studied 361(61.81\%) nerves passed away from the ligament, 182(31.61\%) passed through the ligament and 41(7.02\%) deep to the ligament [12]. Our study has shown similar findings [Table No.2].

Clinical Relevance: Injury to the recurrent laryngeal nerves during thyroid surgeries can be life threatening event and may change the lifestyle of patients completely[13]. Some procedures that may result in injury to the RLNs are thyroidectomies, excision of Zenker's diverticulum, esophagectomies, tracheoplasties, radical neck dissections, correction of patent ductus arteriosum, mediastinascopy and others[14].

The inferior thyroid artery has been described as an important land mark for identifying the RLNs. Deliberate dissection and fastidious hemostasis are essential to prevent RLN injuries and also to prevent post operative bleeding and hematoma formation [15]. 
The idea that the RLN is more frequently posterior to the ITA, and moreover, expecting this relationship to repeat on both sides, gives the surgeon a false sense of security.

If the nerve lies anterior to, or between the branches of ITA, the withdrawl of the thyroid gland from its stream bed results in the withdrawl of the nerve, with injury being more likely than when the nerve is posterior to ITA [3].

Understanding of this concept is essential, especially when the surgeon must perform a total thyroidectomy. In these cases, some authors prefer to ligate the ITA and its branches next to glandular parenchyma, without approaching its trunk, in order not to include the RLN and at the same time, not to compromise the irrigation of the parathyroids [16].

Common site of injury to RLN is close to the berry's ligament. This can be due to excessive traction, nerve getting caught within ligatures, nerve being injured due to electo- coagulation. Inferior laryngeal artery lies posterior to RLN in this area. Bleeders from berry's ligament should not be clamped blindly before identifying the RLN.

At the level of middle third of the gland the RLN is situated close to the capsule of the gland. In cases of pathological enlargement of thyroid glands, this nerve may be enclosed within the thyroid capsule itself before entering the larynx. It is more prone for injury in large swellings involving thyroid gland.

Medial retraction of thyroid lobe makes the nerve more vulnerable during thyroid surgeries. This maneuver stretches the ITA and its branches displacing the nerve anteriorly in the tracheo-oesophageal groove exposing it to danger.

RLN can also be damaged if its blood supply is compromised during surgery. The blood supply to the RLN comes from the inferior laryngeal branch of ITA. It is always prudent to ligate the ITA closer to the gland after it has given off the branch to the RLN [17].

\section{Conclusion}

Proper dissection and anatomical identification of RLN and all its branches is very important and the surgeon must be knowledgeable about the variations in the neurovascular anatomy of the thyroid gland to prevent complications of surgery.

\section{Acknowledgements}

Authors acknowledge the immense help received from the scholars whose articles are cited and included in references of this manuscript. The authors are also grateful to author/editors/publishers of all those articles, journals and books from where the literature for this article has been reviewed and discussed.

\section{References:}

[1]. Walsh J. Galens discovery. Ann Med History 1926;8:176-184.

[2]. Gray SW, Skandalakis JE, Akin JT- Embryological consideretions of thyroid surgery: developmental anatomy of the thyroid, parathyroid and the recurrent laryngeal nerve. Am surg. 1976;42:621-628.

[3]. Skandalakis JE, DrouLiasc, Harlaftis N et al. The recurrent laryngeal nerve. Am Surg 1976; 42: 629-634.

[4]. SiMON MM- Recurrent laryngeal nerve in thyroid surgery- triangle for its recognition and protection. Amer J Surg 1943; 60(2):212-220

[5]. LAHEY FH and Hoover WB - Injuries to the recurrent laryngeal nerve in thyroid operations. Ann surg 1938;108: 545-562.

[6]. Lekacos NL, Tzardis PJ, Sfikakis PJ, Patoulis SD, Restos SD. Course of recurrent laryngeal nerve to the inferior thyroid artery and the Suspensory ligament of berry.Int. Surg. 1992;77(4):287-8 Dec.

[7]. Hunt PS, Poole M, Reeve Ts A reappraisal of the surgical anatomy of the thyroid and parathyroid glands. Brit.Jn.Surg 1968;55:(1) 63-66.

[8]. David.D.Berlin. The recurrent laryngeal nerves in total ablation of normal thyroid gland. An anatomical and surgical, Study. Surg. Gynecol.Obst. 1935; 60: 19-26.

[9]. Bachhuber CA, Complications of thyroid surgery. Anatomy of Recurrent laryngeal nerve, middle thyroid vein and inferior thyroid artery. Am. Jn. Surg. 1943: 60: 96-100.

[10]. C.K Leow and A.J Webb, 'The lateral thyroid ligament of Berry", International Surgery, vol.83, no.1.PP. 75-78, 1998.

[11]. T.R Kumar, P.V Pradeep and M.Raghavan, "Bilobar thyroid agenesis presenting with adenomatous is thmus and hypothyroidism in a 13 year old girl; a case report", Journal of pediatric sciences, vol.2,no.2, article e 21, 2010.

[12]. P.V Pradeep, B.Jayashree, and SKandha S, Harshita- A closer look at Laryngeal nerves during thyroid surgery: A descriptive study of 584 nerves. Anatomy Research International volume 2012, Article ID 490390; 1 to8.

[13]. Pisanu A, Piu S, Cois A, Uccheddu A. Hypocalcemia following total thyroidectomy: early factors predicting long term outcome. G chir 2005; 26(4): 131-4.

[14]. TiTche LL - causes of recurrent laryngeal nerve paralysis Arch otolaryngol 1976;102: 259-261.

[15]. AvvaS G, Dubner S, Heller KS. Re-operation for bleeding after thyroidectomy and parathyroidectomy. Head Neck 2001; 23(7): 544-6

[16]. Cagnol G, Santini J and Demard F- Anatomy of the recurrent nerve-descriptive anatomy, trajectory and relationships, variations. Acta otohinolaryngol Belg 1987: 41(5): 821-827

[17]. Balasubramanian thiagarajan, preventing nerve damage during thyroid surgeries web med central otorhinolaryngology 2012; 3(4): WM coo3260. 
Fig. No.1 Showing: Left RLN dorsal to Left ITA

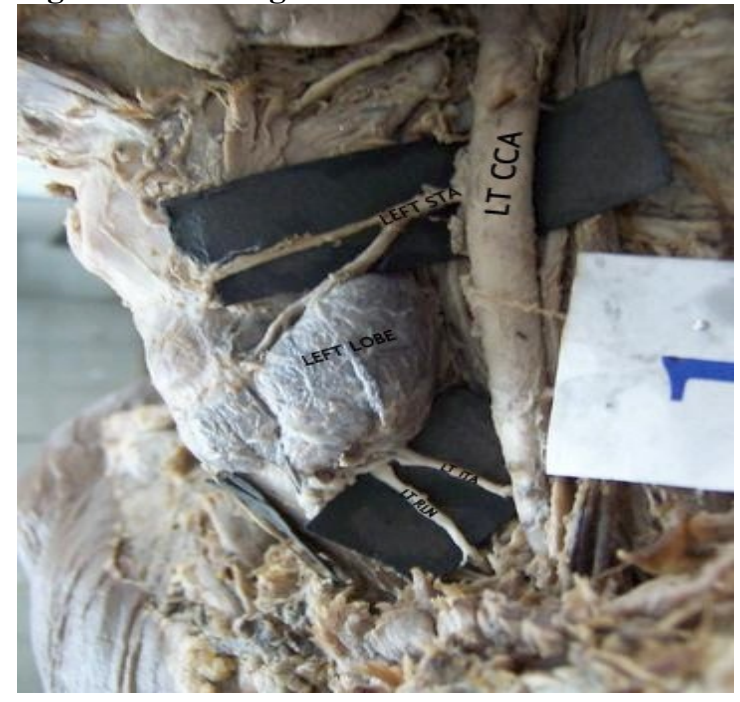

